\title{
Similarities and Differences in Theory of Mind Responses of Patients With Anorexia Nervosa With and Without Autistic Features
}

\author{
Felicity Sedgewick ${ }^{1,2}$, Jenni Leppanen ${ }^{1}$, Faith Goh $^{1}$, Hannah Hayward ${ }^{3}$, Francesca Happé \\ and Kate Tchanturia ${ }^{1,5,6 *}$ \\ 'Institute of Psychiatry, Psychology and Neuroscience, Psychological Medicine, King's College London, London, United Kingdom, \\ ${ }^{2}$ School of Education, University of Bristol, Bristol, United Kingdom, ${ }^{3}$ Department of Forensic and Neurodevelopmental \\ Sciences, Institute of Psychiatry, Psychology and Neuroscience, King's College London, London, United Kingdom, \\ ${ }^{4}$ Medical Research Council (MRC) Social, Genetic and Developmental Psychiatry (SGDP) Centre, King's College London, \\ London, United Kingdom, ${ }^{5}$ South London and Maudsley NHS Trust EDU, London, United Kingdom, ${ }^{6}$ Psychology Department, Illia \\ State University, Tbilisi, Georgia
}

OPEN ACCESS

Edited by: Ute Krügel, Leipzig University, Germany

Reviewed by: Frank M. Schmidt, Universitätsklinikum Leipzig, Germany Juliane Minkwitz, Max Planck Institute of Psychiatry (MPI), Germany

${ }^{*}$ Correspondence: Kate Tchanturia kate.tchanturia@kcl.ac.uk

Specialty section:

This article was submitted to Psychosomatic Medicine, a section of the journal Frontiers in Psychiatry

Received: 01 March 2019 Accepted: 24 April 2019 Published: 08 May 2019

Citation:

Sedgewick F, Leppanen J, Goh F, Hayward H, Happe F and Tchanturia K (2019) Similarities and Differences in Theory of Mind Responses of Patients With Anorexia Nervosa With and

Without Autistic Features.

Front. Psychiatry 10:318.

doi: 10.3389/fpsyt.2019.00318
Theory of Mind (ToM) is the ability to understand and represent mental states of others, a skill that plays a key role in how we interact with people around us. Difficulties with ToM have been posited as an underlying mechanism for autism and implicated in difficulties faced by those with anorexia nervosa (AN). This study examined, both quantitatively and qualitatively, the responses of women between the ages of 14 and 25 years on the Frith-Happé Triangle Animations, a well-validated test of ToM. Participants were split into healthy controls (HCs), AN patients (AN), and AN patients with high levels of autistic features (AN+ASF). We found no significant quantitative differences between groups in performance on the task. Qualitatively, there were differences between groups such that AN patients, especially those in the AN+ASF group, were more focused on describing the videos than creating narratives, were more negative in their interpretations, and were much more anxious about their performance. These qualitative differences have clinical implications, including that not all AN patients with autistic features should be assumed to have difficulties with ToM.

Keywords: eating disorders, autism spectrum disorders, women, Theory of Mind, emotional valence

\section{INTRODUCTION}

Theory of Mind (ToM) is the ability to represent mental states, such as beliefs and intentions, in order to predict and explain people's behavior $(1,2)$. ToM difficulties have been theorized as an explanatory mechanism for the social difficulties that are a defining diagnostic criteria for autism spectrum disorder (3). Large numbers of studies, using a range of ToM tasks, have consistently found that autistic people score lower than neurotypical counterparts in terms of their ability to extrapolate the mental states of characters [e.g., Refs. (4-6)]. A steady accumulation of evidence has shown that people with anorexia nervosa (AN) show similarities in cognitive profile to autistic individuals (7-9). Cognitive features such as poor flexibility and detail-focus (or weak "central coherence") have been documented in both AN and autistic groups relative to healthy controls $(\mathrm{HCs})(9,10)$. People with AN and autistic people appear also to share deficits in social-emotional functioning, including ToM (11). A recent review of the literature comparing autistic people and those with AN (12) reported a number of 
similar difficulties, particularly in complex social situation tasks. Other meta-analyses have suggested that those with AN may have difficulties empathizing with fictional characters in ways similar to those seen in autistic people (13). Cognitive and social-emotional difficulties have been suggested to fuel the illness progression in $\mathrm{AN}$ by increasing isolation and making it difficult to see the big picture or change their thinking around food and exercise (14).

Although there is a solid body of research suggesting links between autism and anorexia going back to the 1980s $(15,16)$, most research to date on ToM in AN has not considered the potential influence of autistic features. This means that those differences and difficulties identified in previous work cannot confidently be associated with AN itself, rather than co-occurring autistic features-something that is an issue, as there is evidence that up to $23 \%$ of women with AN may also be autistic (17).

Therefore, we aimed to investigate whether AN patients who reported high levels of autistic features and those who reported low levels of autistic features differed in ToM ability, and also whether their ToM performance differed from that of HCs, either quantitatively or qualitatively. We expected that those with high levels of autistic features would perform poorly on ToM tasks, similarly to autistic participants in other studies, but that AN participants with low levels of autistic features would perform similarly to HC participants.

\section{METHODS}

\section{Participants}

Data from 57 women between 14 and 25 years old were included in this analysis. Participants with AN and high levels of autistic features [scoring above 6 on the Autism Quotient-10 item version (AQ-10)] were first identified from a larger dataset (BEACON Study, MRC-MRF MR/R004595/1) of 171 participants, resulting in 17 participants meeting criteria. Matched samples of HCs and those with AN and low autistic traits were then identified from within the same dataset and included in this study.

Three groups [HCs, patients with AN with low levels of autistic features, and patients with anorexia and high levels of autistic features $(\mathrm{AN}+\mathrm{ASF})]$ were matched in terms of age and IQ, and each group had a similar ethnic makeup (see Table 1). There was a significant difference between the $\mathrm{HC}$ participants and all $\mathrm{AN}$ participants on BMI, all $p$ 's $<0.001$, but no difference between the AN and AN+ASF groups. Participants were recruited from a range of clinical and community sites across London under ethical approval from the London-Surrey Research Ethics Committee (17/LO/2071). Written informed consent was obtained from all participants, and written informed consent was obtained from the parents of all participants under the age of 16. All AN participants had current clinical diagnoses of AN according to the criteria of the Diagnostic and Statistical Manual-5th edition (3).

To investigate the impact of autistic features, the AN group was split into those with high-autistic (AN+ASF) and low-autistic features (AN), as measured on the AQ-10 (18). Those who scored 6 or more on the AQ-10 were categorized as AN+ASF, using the cutoff for likely autism suggested by the authors of the measure (18), and those with a score of 3 or less were categorized as AN only.
TABLE 1 | Demographic information about participants by group.

\begin{tabular}{lccc}
\hline & HC & AN & AN+ASF \\
\hline $\mathrm{n}$ & 20 & 20 & 17 \\
Age & & & \\
Range & $14.24-24.21$ & $14.43-25.06$ & $14.00-23.26$ \\
$\mathrm{M}(\mathrm{SD})$ & $19.16(2.73)$ & $19.41(3.40)$ & $18.62(2.51)$ \\
\hline IQ & & & \\
Range & $101.27-127.70$ & $93.83-125.22$ & $102.92-115.31$ \\
M (SD) & $112.09(7.64)$ & $110.71(7.86)$ & $109.22(3.57)$ \\
\hline BMl & & & \\
Range & $18.26-26.81$ & $16.00-23.43$ & $14.98-25.16$ \\
M (SD) & $21.54(2.67)$ & $18.04(1.80)$ & $18.59(2.83)$ \\
\hline Ethnicity & $15(75)$ & & $16(94.12)$ \\
White $n(\%)$ & $2(10)$ & $19(95)$ & $0(0)$ \\
Black n (\%) & $1(5)$ & $0(0)$ & $1(5.88)$ \\
Asian n (\%) & $1(5)$ & $0(0)$ & $0(0)$ \\
Latinx n (\%) & $1(5)$ &
\end{tabular}

$H C$, healthy control; $A N$, anorexia nervosa patients; $A N+A S F, A N$ patients with high levels of autistic features.

We did not include participants who scored 4 or 5 in this study due to their being close to the cutoff score, as they may be among those women who are "missed" by diagnostic measures due to presenting in a non-stereotypical manner (19). HC participants were all subject to a screening call prior to taking part to ensure that they had no eating disorder past or present, and all scored less than 2 on the AQ-10 and therefore were considered a valid comparison group, without either AN or autism.

\section{Measures}

AQ-10: The Autism Quotient-10 item version (18) is a 10 -item questionnaire assessing autistic symptomatology. Participants respond on a four-point Likert scale, from "strongly agree" to "strongly disagree," and items are scored either 1 or 0 , depending on the direction of the endorsement. This results in a maximum score of 10 for the measure, and 6 is used as the threshold to indicate potential autism (18).

Theory of Mind: The Frith-Happé Triangle Animations (20, 21) are a series of 10 short silent animations (each 30-40 s long) showing two triangles moving. In two videos, the triangles move at random and do not interact. In four videos, the triangles move in a simple or goal-oriented manner, for example, pushing each other back and forth. In the other four videos, the triangles move in a way that can be interpreted as a complex interaction, such as one triangle encouraging the other to leave an enclosure. These three categories of video-Random, Goal-Oriented, and Complex/ ToM-are designed to elicit different levels of ToM description from participants, who are asked to narrate the videos as they appeared on screen in line with European Autism Interventions - A Multicentre Study for Developing New Medications (EU-AIMS) methodology (22). Answers are then scored 0-2 for Accuracy and 0-2 for Mental State Terms and summed for each video type. Transcripts of participant responses allow for both quantitative and qualitative analysis. The Frith-Happé Triangle Animations have previously been used to assess ToM ability in clinical groups including autism (e.g., 23) and anorexia $(24,25)$. 
The narrative responses were recorded and transcribed for further quantitative and qualitative analysis. Transcription was conducted by one of the authors (FS) who is a native English speaker and was checked for reliability by two other authors (JL and FG). Any disagreements regarding the transcription were brought to the whole team for discussion.

EDE-Q: The Eating Disorder Examination Self-Report (26)-Questionnaire is a 36-item self-report questionnaire assessing eating disorder psychopathology over the past 28 days. Suggested clinical cutoff for the EDE-Q is 2.3 (27).

HADS: The Hospital Anxiety and Depression Scale (28) is a 14-item self-report questionnaire assessing levels of anxiety and depression over the past 2 weeks. Suggested clinical cutoff for the HADS is 8 or above on either subscale or 10 or above on the subscales combined (29).

\section{General Procedure}

Participants were all seen at the university as part of a larger study (BEACON Study, MRC-MRF MR/R004595/1). Participants completed demographic information, the EDE-Q, and the HADS as part of online questionnaires. The larger testing session lasted approximately $3 \mathrm{~h}$, including an autism assessment, a range of neurocognitive tests (including the Frith-Happé Triangle Animations), and a structural and functional MRI scan.

\section{Data Analysis}

Quantitative data were analyzed using R (R Core Team). Group differences in clinical and demographic characteristics were assessed with ANOVA, and Hedges' g was calculated to estimate the effect size. Group differences in ToM task accuracy and mentalizing ability were examined using Poisson regression. Finally, we also explored whether performance on the ToM task was related to eating disorder psychopathology, anxiety and depression, or body mass index (BMI) using Spearman's correlation tests. Due to the large number of exploratory correlation analyses, the p-threshold was adjusted for multiple comparisons using the false discovery rate with $\mathrm{q}=0.05$. P-value less that 0.004 was considered significant.

Qualitative data were collected by recording the spoken responses of participants to the Frith-Happé Triangles and transcribing these verbatim. Thematic analysis of the transcripts was conducted by two authors, one acting as first coder (FS) and the second (FG) carrying out reliability coding of $20 \%$ of the transcripts. Both the first and second coder conducted the thematic analysis blind to both group and codes, so that the second individual was not aware of the themes the first had identified, and the two authors then met to discuss and agree on the results. There were no notable differences between the themes the two authors found in the participants transcripts, and the themes presented below are their consensus coding.

\section{RESULTS}

\section{Quantitative Analyses}

Self-Report Questionnaires

There were significant differences between the groups on EDE-Q Global score, HADS Anxiety, and HADS Depression (see Table 2 for scores). Post hoc t-tests revealed that both AN and AN+ASF groups scored significantly higher than HC participants on the EDE-Q Global score, HADS Anxiety, and HADS Depression. There was no significant difference between the AN and AN+ASF groups on the EDE-Q, $t(35)=-1.85, p=0.07$. There were significant differences between the $\mathrm{AN}$ and $\mathrm{AN}+\mathrm{ASF}$ groups on HADS Anxiety, $t(35)=-4.21, p<0.001$, and HADS Depression,

TABLE 2 | Scores on mental health measures by group.

\begin{tabular}{|c|c|c|c|c|c|}
\hline & $\mathrm{HC}$ & AN & $A N+A S F$ & $\begin{array}{c}\text { F-statistic } \\
\text { p-value }\end{array}$ & $\begin{array}{c}\text { Hedges' g ES } \\
{[95 \% \mathrm{Cl}]}\end{array}$ \\
\hline $\begin{array}{l}\text { AQ-10 } \\
\text { Range } \\
M(S D)\end{array}$ & $\begin{array}{c}0-2 \\
1.00(1.00)\end{array}$ & $\begin{array}{c}1-2 \\
2.00(1.00)\end{array}$ & $\begin{array}{c}6-10 \\
7.00(1.00)\end{array}$ & $\begin{array}{c}F(2)=268.03 \\
p<0.001^{*}\end{array}$ & $\begin{array}{c}\text { HC vs. AN: }-0.76, \\
{[-1.42,-0.09]} \\
\text { HC vs. AN+ASF: }-5.58 \\
{[-7.06,-4.10]} \\
\text { AN vs. AN+ASF: }-5.48 \\
{[-6.93,-4.02]}\end{array}$ \\
\hline $\begin{array}{l}\text { EDE-Q Global } \\
\text { Range } \\
\text { M (SD) }\end{array}$ & $\begin{array}{c}0-0.73 \\
0.23(0.20)\end{array}$ & $\begin{array}{l}0.28-5.12 \\
2.63(1.52)\end{array}$ & $\begin{array}{l}0.35-5.12 \\
3.54(1.44)\end{array}$ & $\begin{array}{l}F(2)=38.37 \\
p=<0.001^{\star}\end{array}$ & $\begin{array}{c}\text { HC vs. AN: }-2.17, \\
{[-2.98,-1.36]} \\
\text { HC vs. AN+ASF: }-3.03, \\
\text { [-4.01, -2.05] } \\
\text { AN vs. AN+ASF: }-0.60, \\
{[-1.28,0.09]}\end{array}$ \\
\hline $\begin{array}{l}\text { HADS Total } \\
\text { Range } \\
\text { M (SD) }\end{array}$ & $\begin{array}{c}1-21 \\
8.15(5.76)\end{array}$ & $\begin{array}{c}3-27 \\
14.65(5.67)\end{array}$ & $\begin{array}{c}14-42 \\
24.12(6.74)\end{array}$ & $\begin{array}{l}F(2)=32.29 \\
p=<0.001^{\star}\end{array}$ & $\begin{array}{c}\text { HC vs. AN: }-1.11, \\
{[-180,-0.43]} \\
\text { HC vs. AN+ASF: } 2.48, \\
{[-3.37,-1.59]} \\
\text { AN vs. AN+ASF: }-1.48, \\
{[-2.23,-0.72]}\end{array}$ \\
\hline
\end{tabular}

AQ-10, Autism Quotient-10 item version; EDE-Q, Eating Disorder Examination Self-Report Questionnaire; HADS, Hospital Anxiety and Depression Scale. ${ }^{*}$ Denotes a significant result. 
$t(35)=-3.81, p=0.001$, such that AN+ASF participants were more anxious and more depressed than AN participants. There were no correlations between AQ-10 and BMI in the HC group $(\rho=0.13, p=0.581)$, AN group $(\rho=0.00, p=1.00)$, or the AN+ASF group $(\rho=-0.19, p=0.475)$. There were no correlations between AQ-10 score and EDE-Q score in the HC group $(\rho=-0.02$, $p=0.949)$, AN group $(\rho=0.17, p=0.485)$, or AN+ASF group $(\rho=-0.39, p=0.117)$. There was also no significant correlation between AQ-10 scores and HADS scores within the HC group ( $\rho=-0.04, p=0.862)$, the AN group $(\rho=-0.022, p=0.355)$, or the AN+ASF group $(\rho=0.11, p=0.675)$.

\section{Theory of Mind: Accuracy}

On Accuracy, there was no significant difference between the three groups on either the Random, the Goal-Oriented, or the ToM animations (see Table 3 for scores).

\section{Theory of Mind: Mental State}

On Mental State, there were no significant differences between the three groups in any of the video conditions (Random, GoalOriented, Complex, Total; see Table 3 for scores).

\section{Clinical Measures and Theory of Mind}

We explored whether levels of eating disorder behaviors, anxiety and depression, autistic features, and BMI had an impact on either accuracy or on number of mental state terms, negative, and positive terms participants used in their narrations. Due to there being no significant group differences between the $\mathrm{HC}, \mathrm{AN}$, and AN+ASF, all participants were included in the exploratory correlation analyses. There were no significant correlations between Accuracy or Mental State Language and any clinical measures across all participants (see Table 4).

\section{Qualitative Analysis}

Qualitatively, there were notable differences between the three groups. These differences can be characterized as coming under the themes of detail focus, negative interpretation bias, inaccurate emotional labels, and anxiety and are visualized in Figure 1.

Detail focus. The most obvious difference between the patients and the HC participants was their different level of focus on the detailed geometry and movement in the videos. AN patients, particularly those with high AQ scores, gave greater focus to precisely where items were on the screen, for example, saying "the smaller box with the open side is now in the bottom right hand corner" (AN+ASF; Chasing) or "the little triangle is moving in a clockwise direction and the bigger triangle in an anti-clockwise direction" (AN; Bouncing). While some HC participants also described where the triangles were throughout the videos, these descriptions tended to relate their positioning to each other- "the little one is above the big one" (HC; Floating). In contrast, AN participants often described the triangles independently of each other, even in interaction videos- "the little one is in the box and the big one is going in and out of the box" (AN+ASF: Coaxing).

AN participants also placed more emphasis on describing the appearance of the triangles, rather than creating a narrative around their actions. For example, the patient group said things like "the lines keep getting smoother" (AN+ASF; Bouncing), "they are drawn in graphite" (AN; Surprise), and "they've squashed so they aren't isosceles triangles anymore, they're more like obtuse triangles" (AN+ASF; Fighting). While some HC participants did describe the layout of the screen- "there's a smaller box with an open side" (HC; Coaxing) — this was far less common and was usually instrumental to describing the action, rather than being a standalone comment. In the above example, the sentence was finished by adding "and the triangles are inside

TABLE 3 | Accuracy and Mental State Language scores by group and video type.

\begin{tabular}{|c|c|c|c|c|c|}
\hline & $\mathrm{HC}$ & AN & $A N+A S F$ & $\begin{array}{c}X^{2} \text {-statistic } \\
p \text {-value }\end{array}$ & Cramer's $V$ \\
\hline \multicolumn{6}{|l|}{ Accuracy } \\
\hline Random Range & $2-4$ & $0-4$ & $0-4$ & $X^{2}(2)=0.65$ & 0.08 \\
\hline Median (IQR) & $3.0(2.0)$ & $3.0(2.0)$ & $4.0(1.5)$ & $p=0.721$ & \\
\hline \multicolumn{6}{|l|}{ Accuracy } \\
\hline Goal-oriented Range & $1-9$ & $2-7$ & 3-7 & $X^{2}(2)=0.45$ & 0.06 \\
\hline Median (IQR) & $5.0(2.0)$ & $6.0(1.0)$ & $5.0(1.5)$ & $p=0.799$ & \\
\hline \multicolumn{6}{|l|}{ Accuracy } \\
\hline Complex Range & $2-7$ & $1-6$ & $0-7$ & $X^{2}(2)=1.03$ & 0.10 \\
\hline Median (IQR) & $4.0(0.5)$ & $4.0(2.0)$ & $3.0(2.0)$ & $p=0.599$ & \\
\hline \multicolumn{6}{|l|}{ Mental State } \\
\hline Random Range & $0-1$ & 0-2 & $0-1$ & $X^{2}(2)=2.30$ & 0.14 \\
\hline Median (IQR) & $0.0(0.0)$ & $0.0(1.0)$ & $0.0(0.0)$ & $p=0.317$ & \\
\hline \multicolumn{6}{|l|}{ Mental State } \\
\hline Goal-oriented Range & $0-2$ & $0-4$ & 0-3 & $X^{2}(2)=1.12$ & 0.10 \\
\hline Median (IQR) & $1.0(2.0)$ & $0.0(2.0)$ & $0.0(1.0)$ & $p=0.572$ & \\
\hline \multicolumn{6}{|l|}{ Mental State } \\
\hline Complex Range & $0-7$ & $0-7$ & $0-6$ & $X^{2}(2)=5.89$ & 0.28 \\
\hline Median (IQR) & $3.0(1.5)$ & $3.5(2.0)$ & $2.0(3.0)$ & $p=0.053$ & \\
\hline
\end{tabular}

Italicisation is a convention of the field. 
TABLE 4 | Correlations between self-reported mental health and Theory of Mind.

\begin{tabular}{lcccc}
\hline & Accuracy & $\begin{array}{c}\text { Mental State } \\
\text { Language }\end{array}$ & $\begin{array}{c}\text { Negative } \\
\text { terms }\end{array}$ & $\begin{array}{c}\text { Positive } \\
\text { terms }\end{array}$ \\
\hline EDE-Q Total & $\rho=-0.20$, & $\rho=-0.19$, & $\rho=-0.01$, & $\rho=0.11$, \\
& $\rho=0.135$ & $p=0.155$ & $p=0.932$ & $p=0.409$ \\
\hline HADS Total & $\rho=-0.18$, & $\rho=-0.17$, & $\rho=-0.01$, & $\rho=0.03$, \\
& $p=0.190$ & $p=0.195$ & $p=0.949$ & $p=0.813$ \\
\hline AQ-10 Total & $\rho=-0.09$, & $\rho=-0.13$, & $\rho=-0.12$, & $\rho=-0.06$, \\
& $p=0.518$ & $p=0.320$ & $p=0.354$ & $p=0.614$ \\
\hline BMI & $\rho=0.23$, & $\rho=0.29$, & $\rho=0.22$, & $\rho=0.21$, \\
& $p=0.091$ & $p=0.030$ & $p=0.107$ & $p=0.125$ \\
\hline
\end{tabular}

it" (HC; Coaxing), whereas AN participants tended to focus on describing the physical appearance of the objects on the screen rather than providing a narrative.

Negative interpretation bias. Another key qualitative difference between the groups was the prevalence of negative emotional words. Both AN and AN+ASF participants used far more negative terms and gave more negative interpretations of the triangles' movements than HC participants, describing them as "crying," "fighting," and "angry" more often than HC participants. They gave more involved descriptions of these negative emotions and cognitions than for positive ones- "he's hiding in the corner shaking 'cos he's scared and trapped" (AN; Seduction) compared to "they're happy they've found each other" (HC; Surprise). AN+ASF participants were most likely to give these negative interpretations, with AN participants representing a "mid-point" between the AN+ASF and HC groups in terms of the negative emotions they assigned to the triangles. Rather than the negative emotions common among AN+ASF participants, AN and HC participants mostly used emotionally neutral terms - "wants to get out," "is looking for the other one," or "tries to get in the box." These terms all imply a mental state or goal of the character, without giving much emotional weight to those mental states and desires.

Beyond the individual negative terms used by the patient group, the AN+ASF group gave much more negative interpretations of the videos overall. For example, one participant described the Coaxing scenario (where the big triangle pulls the little one out of the "house" to spin and play in the "garden") as the two triangles fighting and the "small one crying in the corner because it was scared," then spinning because it was "scared and confused." This is both inaccurate and a negative way of seeing the situation, which is supposed to show two characters having fun. More AN+ASF participants gave these unhappy interpretations of either neutral or positive scenarios compared to AN and HC participants who were more positive.

As a specific example of this negative interpretation, the "Surprise" video is supposed to represent a game of "knock and run" - the larger triangle is inside the "house" by itself, and then the smaller one enters and bumps several times on the "door" ("knocking"). The larger triangle opens the door (side of the box), comes part way out, and bends from side to side, "looking" for the one who knocked, while the small one "hides" behind the door. The large triangle goes back inside, and the sequence is repeated. On the third time of "knocking," the small triangle "surprises" the large one by coming out from behind the door, and then the two triangles enter the box together and spin (intended to represent hugging or being pleased to see each other).

The authors of the animations expected participants to see this as a game of knock and run between a grandmother and grandson (or similar characters), and to give answers relating to the game and the small triangle surprising or tricking the large triangle. Instead, AN participants frequently interpreted the scenario negatively. Common narratives were that the large triangle was "trapped" in the box and "can't get out," or that the small triangle was "locking it in," "turning a switch to keep it in," or that the large triangle "is locking [the small one] out when it wants to get in." When they used ToM terms to describe how the triangles felt about the scenario, they used terms associated with negative emotions such as "sad," "worried," and "feels unwanted," rather than the positive emotions of surprise and joy that the authors intended to be seen in the video. This was true for all AN participants, rather than being affected by ASF status.

Inaccurate emotional labels. Linked to this negative interpretation bias, participants often misinterpreted the emotional valence of videos, seeing them as negative when they were neutral or positive in nature. Participants generally used more negative

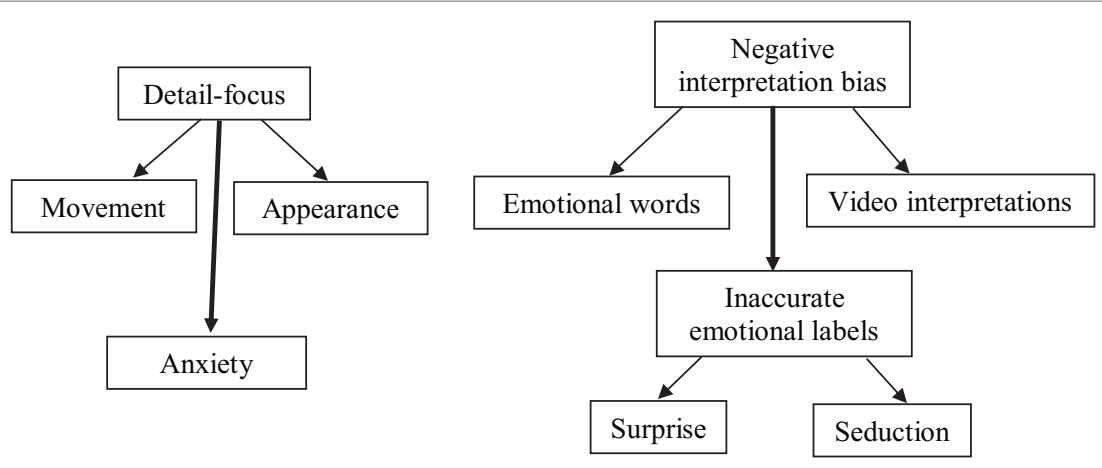

FIGURE 1 | Qualitative map of themes arising from narrations of the Frith-Happé Triangle Animations. 
than positive emotional labels when narrating the videos, and the negative words they used were more intense than the positive terms, i.e., "[the triangle is] shaking and scared" or "[it looks like it's been] murdered" compared to "[the big one is] pleased" and "[they're having] fun."

Interestingly, the Seduction scenario was also interpreted very differently by participants than intended according to the scoring manual. The interpretation given in the scoring manual is that of a princess tricking a guard into letting her escape-a situation that could be interpreted negatively, although there is a happy ending. This video, which would seem to lend itself to the negative interpretations shown by AN+ASF participants elsewhere, did not elicit the same kind of strong negative responses. Instead, $\mathrm{AN}+\mathrm{ASF}$ participants tended to simply describe the geometry of this video, as discussed above. In contrast, $\mathrm{AN}$ and $\mathrm{HC}$ participants tended to describe the small triangle as "escaping" or "running away," and the large triangle as "confused"-terms that imply mental states being attributed to the characters, even if they were presented in terms with neutral emotional valence.

Anxiety. Another notable pattern, possibly linked to the detailfocus discussed above, was that the patient group was much more likely than HC participants to ask questions of the examiner during the administration of the task, focused on making sure that they were getting things "right" or performing as expected. Several AN and AN+ASF participants asked questions such as "Is that what you wanted?", "Is that right?," or "Have I got it all?" Participants in both the AN+ASF and AN groups were notably more anxious about saying the right thing, giving the right interpretation, and simply whether they were completing the task correctly than HC participants were. This desire to give "perfect" responses, and the accompanying anxiety about not doing so, was not present in HC participants.

\section{DISCUSSION}

The findings of this study suggest that while there were no statistically significant quantitative differences between $\mathrm{HC}$ participants, AN participants, and AN+ASF participants on the Frith-Happé Triangle Animations, there are some interesting qualitative differences. This suggests that people with AN may not have significant difficulties in ToM, regardless of their level of self-reported autistic features. Although several studies have reported significantly reduced ToM in people with AN using a variety of tasks (12), more recently a few larger studies using a variety of different measures have found no group differences (24). Together, these findings suggest that women with AN may have specific social-emotional difficulties that may not extend to explicit labeling and recognition of emotions and mental states, but may rather be reflective of more subtle qualitative difficulties, such as interpretation biases.

Contrary to our hypothesis, there were no significant differences between the high-AQ and low-AQ groups in terms of Accuracy or Mental State Language, regardless of AN status. It should be pointed out, however, that scoring above cutoff on the AQ-10 is distinctly different to receiving a clinical diagnosis of being autistic, meaning that these individuals may not be as similar in performance on the task to autistic people as might be expected. Further to this, the AQ-10 is a basic screening tool and, while widely used, has been shown to be less reliable than the longer versions of the AQ, such as the AQ-50 or AQ-28 $(30,31)$, or other self-report questionnaires such as the Social Responsiveness Scale-2 ${ }^{\text {nd }}$ Edition (32). However, we can be confident in this sample that the AQ-10 was reflective of autistic features rather than mental health issues, as there was no correlation between AQ-10 score and HADS score in any group.

It is also worth noting that previous research that has supported the effectiveness of the Frith-Happé Triangle Animations as a measure of ToM ability, and as revealing differences between autistic and neurotypical groups, has had mainly male participants. The non-significant quantitative findings in this study may therefore actually represent a gender difference according to the scoring method that focuses on accuracy in explicit labeling and recognition. What little evidence there is as to gender differences in ToM ability suggests that girls score more highly than boys (20, 21, 33). Among neurotypical children, one study found that 3- to 5-year-old non-autistic girls scored better on ToM tasks than non-autistic boys (34) on the Sally-Anne False Belief task, and this gender difference is also seen in late childhood (35). Most work that finds autistic people struggle with ToM has used majority-male participant samples, as is the case for much autism research (36). This means that we know very little about ToM ability in autistic girls and women, or in girls and women with high levels of autistic features. There is, to date, little research on the ToM skills of older girls and women with eating disorders, regardless of their autism status, a lack that this paper seeks to somewhat redress. Future work should seek matched groups of males with $\mathrm{AN}$ and autistic males and females without AN in order to establish the true nature of the similarities and differences in ToM in $\mathrm{AN}$ and any potential relationship to autistic features. It is also worth noting that all participants in this sample had relatively high IQ scores, something that may ameliorate difficulties with ToM traditionally seen among autistic individuals.

The findings of this research suggest that the Frith-Happé Triangle Animations (20) may not be the most sensitive measure of ToM to use in a female neurotypical population, similar to other research findings (37). All women in this study scored similarly on both Accuracy and Mental State, regardless of levels of self-reported autistic features. Also, most participants did not create story-like narratives for the videos, instead describing the screen and the movements of the triangles, something that may have been induced in part by the direction to describe the video as it was happening. This suggests that although the task seeks to examine ToM skill-looking at the intentions and motives assigned to the two triangles-the administration instructions do not explicitly ask for this, and therefore, some participants may not show their actual level of ToM skill. While some participants did create stories and use a range of ToM terms, most did not create complex narratives, instead giving individual mentalizing terms or inconsistently using designating the triangles as characters.

The qualitative findings of this study echo those of other work that has suggested similarities between the social and 
cognitive experiences of autistic people and patients with AN. The differences in the negative interpretations of the scenarios between $\mathrm{HC} / \mathrm{AN}$ participants and $\mathrm{AN}+\mathrm{ASF}$ participants were clear. That AN+ASF participants generally use more negative terms and give more negative interpretations to the FrithHappé Animations aligns with previous findings of negative interpretation bias in AN (38-40). This is further evidence that AN patients with co-occurring autistic traits may need extra or individually tailored support in their treatment programs, as social support can be crucial to recovery (41-43), but if they are consistently interpreting their social experiences negatively, they may be struggling to access that support. Difficulties with negative interpretation are potentially further exacerbated by the higher levels of depression and anxiety in the AN group, as individuals with depression $(44,45)$ and anxiety $(46,47)$ have been shown to interpret situations negatively. Therefore, patients who have co-occurring $\mathrm{AN}$, depression, and autism may be particularly negative in their views of their social experiences, which creates a self-fulfilling cycle where they interpret a situation negatively and withdraw or react inappropriately; therefore, those around them are less supportive, reinforcing the idea that they do not have social support, and giving the impression that their initial negative interpretation was correct. This interpretation is supported by work showing that those who are "affective deviants," i.e., who react in non-normative ways to social situations, are judged more negatively by those they interact with and are more likely to be avoided by others (48).

Other qualitative work examining emotions in anorexia found that patients had difficulties with emotional expression and negative emotions (49), all of which are also seen among autistic people (50-52). Similarly, patients with AN have been shown to have difficulties with their friendships and social relationships, which predate the onset of their illness $(53,54)$, and challenges with social relationships and imagination are a key diagnostic feature of autism (3). Importantly, recent research has shown that these difficulties are present for autistic women and girls (55-57), meaning that these experiences are directly comparable to those of female AN patients.

The focus of AN participants, particularly AN+ASF participants, on describing the layout of the screen and the movements of the triangles provides qualitative evidence of the detail-oriented processing and weak central coherence that has been previously seen in both those with $\mathrm{AN}(10,58,59)$ and those on the autism spectrum (60-62). It may be that individuals at the intersection of the two conditions, potentially represented by the AN+ASF group, would have that much more of a focus on details rather than the overall narrative. Knowing that this is a potential cognitive profile, as with the tendency to negative interpretation bias, has clinical implications. Detail-oriented processing over global processing is a known factor in many treatment programs designed to support AN recovery, but if a patient also has high levels of autistic features, they may find it particularly difficult to move on from this thinking style, as it may be linked not only to their illness but also to their underlying neurotype. This means that clinical teams would need to adapt treatment approaches to their particular needs and may need to reframe the ways in which these approaches are presented (63).
The fact that AN patients asked far more questions than the $\mathrm{HC}$ participants points to the much higher anxiety levels of the patient group. These higher anxiety levels are borne out in the quantitative as well as qualitative data, with $\mathrm{AN}$ and $\mathrm{AN}+\mathrm{ASF}$ participants being more anxious than HC participants. There is a wealth of research evidencing higher anxiety levels in AN patients $(64,65)$ and autistic people (66-68). The emphasis in these questions on whether participants were "doing the right thing" or "giving the right answer" suggests that $\mathrm{AN}$ and AN+ASF participants were especially anxious about their performance on the task rather than how to complete it, an attitude that may be linked to the high levels of perfectionism seen in those with AN (69-71).

\section{LIMITATIONS}

While there were limitations to this study, such as the small sample size, the number of participants is sufficient for the analyses conducted, as shown by the range and consistency of the group differences identified. In the quantitative analysis, there is, however, the possibility of type II errors when working with a small sample size, as there may be insufficient power to detect a true effect leading to a negative finding. Therefore, larger studies are needed before firm conclusions regarding ToM difficulties in people with AN and ASF can be drawn. Future quantitative studies may also benefit from including a larger sample to assess the impact of eating disorders psychopathology, illness stage, and subtype along with other mental health measures on ToM scores to gain a more holistic picture of social-emotional difficulties in AN.

Another issue is the potential lack of sensitivity of the AQ-10, but it is widely used as a screening measure both clinically $(17,72)$ and in research $(18,73,74)$. The fact that the use of the AQ-10 cutoff did not make a statistically meaningful difference to the outcome measures also suggests that the AQ-10, while quick to administer, may not be the most effective screening tool in a clinical setting, and therefore it may be worth clinical teams taking more time to use more thorough measures. In future work, it will be crucial to have a comparison group of people with $\mathrm{AN}$ who also have clinicianverified autism diagnoses, and a comparison group of autistic people without $\mathrm{AN}$, to more fully examine the impact of autistic features on social-emotional difficulties in AN and place these in the context of autism itself. The all-female nature of this sample is also a limitation of the study. While most ToM work has had majority-male samples, as it comes from the autism field, most AN work has majority-female samples, as these are the people who are most often diagnosed with eating disorders. Conducting research with gender-balanced samples will be important in the future to redress the existing gender imbalance in both autism and eating disorder research and will allow us to more accurately evaluate and describe group- and gender-based differences in these skills.

\section{CONCLUSION}

Overall, the findings of this study suggest that while there may not be quantitative differences in task performance between HCs, AN patients with low-AQ scores and patients with high-AQ scores on the Frith-Happé Triangle Animations test of ToM, the qualitative differences between the groups may have 
clinical implications. The findings also bring into question the assumption that everyone with high levels of autistic features will have difficulties with ToM tasks, highlighting that this may instead be a feature of autism in males rather than females.

\section{DATA AVAILABILITY STATEMENT}

The datasets generated for this study are available on request to the corresponding author.

\section{AUTHOR CONTRIBUTIONS}

FS and JL conducted data collection, analysis, and primary write-up of the manuscript. $\mathrm{HH}$ and $\mathrm{FH}$ contributed to initial research design and editing of the manuscript. FG contributed to data transcription and analysis, and to edits of the manuscript.

\section{REFERENCES}

1. Baron-Cohen S. Theory of mind and autism: a review. Int Rev Res Ment Retard (2000) 23:169-84. doi: 10.1016/S0074-7750(00)80010-5

2. Baron-Cohen S, Leslie AM, Frith U. Does the autistic child have a "theory of mind"? Cognition (1985) 21(1):37-46. doi: 10.1016/0010-0277(85)90022-8

3. American Psychiatric Association. Diagnostic and statistical manual of mental disorders: DSM-5. Washington D.C: American Psychiatric Association (2013). doi: 10.1176/appi.books.9780890425596

4. Baron-Cohen S, Wheelwright S, Hill J, Raste Y, Plumb I. The "Reading the Mind in the Eyes" Test revised version: a study with normal adults, and adults with Asperger syndrome or high-functioning autism. J Child Psychol Psychiatry (2001) 42(2):241-51. doi: 10.1111/1469-7610.00715

5. Happé FGE. An advanced test of theory of mind: understanding of story characters' thoughts and feelings by able autistic, mentally handicapped, and normal children and adults. J Autism Dev Disord (1994) 24(2):129-54. doi: 10.1007/BF02172093

6. Mathersul D, McDonald S, Rushby JA. Understanding advanced theory of mind and empathy in high-functioning adults with autism spectrum disorder. J Clin Exp Neuropsychol (2013) 35(6):655-68. doi: 10.1080/ 13803395.2013.809700

7. Gillberg IC, Gillberg C, Råstam M, Johansson M. The cognitive profile of anorexia nervosa: a comparative study including a community-based sample. Compr Psychiatry (1996) 37(1):23-30. doi: 10.1016/S0010-440X (96)90046-2

8. Lang K, Stahl D, Espie J, Treasure J, Tchanturia K. Set shifting in children and adolescents with anorexia nervosa: an exploratory systematic review and meta-analysis. Int J Eat Disord (2014) 47(4):394-9. doi: 10.1002/ eat. 22235

9. Westwood H, Stahl D, Mandy W, Tchanturia K. The set-shifting profiles of anorexia nervosa and autism spectrum disorder using the Wisconsin Card Sorting Test: a systematic review and meta-analysis. Psychol Med (2016) 46(9):1809-27 doi: 10.1017/S0033291716000581

10. Lang K, Roberts M, Harrison A, Lopez C, Goddard E, Khondoker M, et al. Central coherence in eating disorders: a synthesis of studies using the Rey Osterrieth complex figure test. PLoS One (2016) 11(11):e0165467. doi: 10.1371/journal.pone.0165467

11. Tchanturia K, Happé F, Godley J, Treasure J, Bara-Carril N, Schmidt U. "Theory of mind" in anorexia nervosa. Eur Eat Disord Rev (2004) 12(6):3616. doi: 10.1002/erv.608

12. Leppanen J, Sedgewick F, Treasure J, Tchanturia K. Differences in the Theory of Mind profiles of patients with anorexia nervosa and individuals on the autism spectrum: a meta-analytic review. Neurosci Biobehav Rev (2018) 90:146-63. doi: 10.1016/j.neubiorev.2018.04.009
KT was PI for the project, was awarded the funding which made it possible, was responsible for initial study design and contributed to recruitment and edits of the manuscript.

\section{FUNDING}

All authors would like to acknowledge the MRC-MRF Fund (MR/R004595/1) for making this research possible. KT would also like to acknowledge funding from The Health Foundation (AIMS ID: 1115447).

\section{ACKNOWLEDGMENTS}

KT would like to acknowledge Prof Tracey Wade and the Norman Munn Distinguished Visiting Scholar Award 2017/2018 from Flinders University South Australia.

13. Kerr-Gaffney J, Harrison A, Tchanturia K. Cognitive and affective empathy in eating disorders: a systematic review and meta-analysis. Front Psychiatry (2019). doi: 10.3389/fpsyt.2019.00102

14. Treasure J, Schmidt U. The cognitive-interpersonal maintenance model of anorexia nervosa revisited: a summary of the evidence for cognitive, socioemotional and interpersonal predisposing and perpetuating factors. $J$ Eat Disord (2013) 1(1):13. doi: 10.1186/2050-2974-1-13

15. Gillberg C. Autism and anorexia nervosa: related conditions? Nordisk Psykiatrisk Tidsskrift (1985) 39(4):307-12.

16. Westwood H, Tchanturia K. Autism spectrum disorder in anorexia nervosa: an updated literature review. Curr Psychiatry Rep (2017) 19(7):41

17. Westwood H, Eisler I, Mandy W, Leppanen J, Treasure J, Tchanturia K. Using the autism-spectrum quotient to measure autistic traits in anorexia nervosa: a systematic review and meta-analysis. J Autism Dev Disord (2016) 46(3):964-77. doi: 10.1007/s10803-015-2641-0

18. Allison C, Auyeung B, Baron-Cohen S. Toward brief "red flags" for autism screening: the short autism spectrum quotient and the short quantitative checklist in 1,000 cases and 3,000 controls. J Am Acad Child Adolesc Psychiatry (2012) 51(2):202-12.e7. doi: 10.1016/j.jaac.2011.11.003

19. Gould J, Ashton-Smith J. Missed diagnosis or misdiagnosis? Girls and women on the autism spectrum. Good Autism Practice (GAP) (2011) 12(1):34-41.

20. Abell F, Happé F, Frith U. Do triangles play tricks? Attribution of mental states to animated shapes in normal and abnormal development. Cogn Dev (2000) 15(1):1-16. doi: 10.1016/S0885-2014(00)00014-9

21. Castelli F, Frith C, Happé F, Frith U. Autism, Asperger syndrome and brain mechanisms for the attribution of mental states to animated shapes. Brain (2002) 125(8):1839-49. doi: 10.1093/brain/awf189

22. Loth E, Charman T, Mason L, Tillmann J, Jones EJH, Wooldridge C, et al. The EU-AIMS Longitudinal European Autism Project (LEAP): design and methodologies to identify and validate stratification biomarkers for autism spectrum disorders. Mol Autism (2017) 8(1):24. doi: 10.1186/ s13229-017-0146-8

23. Wilson CE, Happé F, Wheelwright SJ, Ecker C, Lombardo MV, Johnston P, et al. The neuropsychology of male adults with high-functioning autism or Asperger syndrome. Autism Res (2014) 7(5):568-81. doi: 10.1002/aur.1394

24. Bentz M, Jepsen JRM, Pedersen T, Bulik CM, Pedersen L, Pagsberg AK, et al. Impairment of social function in young females with recent-onset anorexia nervosa and recovered individuals. J Adolesc Health (2017) 60(1):23-32. doi: 10.1016/j.jadohealth.2016.08.011

25. Kothari R, Barona M, Treasure J, Micali N. Social cognition in children at familial high-risk of developing an eating disorder. Front Behav Neurosci (2015) 9:208. doi: 10.3389/fnbeh.2015.00208

26. Luce $\mathrm{KH}$, Crowther $\mathrm{JH}$. The reliability of the eating disorder examinationself-report questionnaire version (EDE-Q). Int J Eat Disord (1999) 25(3):349-51. 
27. Mond J, Hay P, Rodgers B, Owen C, Beumont PJ. Validity of the Eating Disorder Examination Questionnaire (EDE-Q) in screening for eating disorders in community samples. Behav Res Ther (2004) 42(5):551-67. doi: 10.1016/S0005-7967(03)00161-X

28. Zigmond AS, Snaith RP. The hospital anxiety and depression scale. Acta Psychiatr Scand (1983) 67(6):361-70. doi: 10.1111/j.1600-0447.1983. tb09716.x

29. Bjelland I, Dahl AA, Haug TT, Neckelmann D. The validity of the Hospital Anxiety and Depression Scale: an updated literature review. J Psychosom Res (2002) 52(2):69-77. doi: 10.1016/S0022-3999(01)00296-3

30. Baron-Cohen S, Wheelwright S, Skinner R, Martin J, Clubley E. The autism-spectrum quotient (AQ): evidence from Asperger syndrome/ high-functioning autism, males and females, scientists and mathematicians. J Autism Dev Disord (2001) 31(1):5-17. doi: 10.1023/ A:1005653411471

31. Hoekstra RA, Vinkhuyzen AAE, Wheelwright S, Bartels M, Boomsma DI, Baron-Cohen S, et al. The construction and validation of an abridged version of the autism-spectrum quotient (AQ-Short). J Autism Dev Disord (2011) 41(5):589-96. doi: 10.1007/s10803-010-1073-0

32. Constantino JN, Gruber CP. Social responsiveness scale-second edition (SRS-2). Torrance: Western Psychological Services (2012).

33. White SJ, Coniston D, Rogers R, Frith U. Developing the Frith-Happé animations: a quick and objective test of Theory of Mind for adults with autism. Autism Res (2011) 4(2):149-54. doi: 10.1002/aur.174

34. Walker S. Gender differences in the relationship between young children's peer-related social competence and individual differences in theory of mind. J Genet Psychol (2005) 166(3):297-312. doi: 10.3200/GNTP.166.3.297-312

35. Devine RT, Hughes C. Silent films and strange stories: theory of mind, gender, and social experiences in middle childhood. Child Dev (2013) 84(3):989-1003. doi: 10.1111/cdev.12017

36. Banach R, Thompson A, Szatmari P, Goldberg J, Tuff L, Zwaigenbaum L, et al. Brief Report: relationship between non-verbal IQ and gender in autism. J Autism Dev Disord (2009) 39(1):188-93. doi: 10.1007/s10803-008-0612-4

37. Brewer N, Young RL, Barnett E. Measuring theory of mind in adults with autism spectrum disorder. J Autism Dev Disord (2017) 47(7):1927-41. doi: 10.1007/s10803-017-3080-x

38. Cardi V, Turton R, Schifano S, Leppanen J, Hirsch CR, Treasure J. Biased interpretation of ambiguous social scenarios in anorexia nervosa. Eur Eat Disord Rev (2017) 25(1):60-4. doi: 10.1002/erv.2493

39. Cooper M. Bias in interpretation of ambiguous scenarios in eating disorders. Behav Res Ther (1997) 35(7):619-26. doi: 10.1016/S0005-7967(97)00021-1

40. Leppanen J, Cardi V, Ng KW, Paloyelis Y, Stein D, Tchanturia K, et al. Effects of intranasal oxytocin on the interpretation and expression of emotions in anorexia nervosa. J Neuroendocrinol (2017) 29(3). doi: $10.1111 /$ jne. 12458

41. McCormack A. Individuals with eating disorders and the use of online support groups as a form of social support. Comput Inform Nurs (2010) 28(1):12-9. doi: 10.1097/NCN.0b013e3181c04b06

42. Tiller JM, Sloane G, Schmidt U, Troop N, Power M, Treasure JL. Social support in patients with anorexia nervosa and bulimia nervosa. Int J Eat Disord (1997) 21(1):31-8. doi: 10.1002/(SICI)1098-108X(199701)21:1<31:: AID-EAT4>3.3.CO;2-N

43. Weaver K, Wuest J, Ciliska D. Understanding women's journey of recovering from anorexia nervosa. Qual Health Res (2005) 15(2):188-206. doi: 10.1177/1049732304270819

44. Hindash AHC, Amir N. Negative interpretation bias in individuals with depressive symptoms. Cognit Ther Res (2012) 36(5):502-11. doi: 10.1007/ s10608-011-9397-4

45. Mogg K, Bradbury KE, Bradley BP. Interpretation of ambiguous information in clinical depression. Behav Res Ther (2006) 44(10):1411-9. doi: 10.1016/j. brat.2005.10.008

46. Amir N, Beard C, Bower E. Interpretation bias and social anxiety. Cognit Ther Res (2005) 29(4):433-3. doi: 10.1007/s10608-005-2834-5

47. Miers AC, Blöte AW, Bögels SM, Westenberg PM. Interpretation bias and social anxiety in adolescents. J Anxiety Disord (2008) 22(8):1462-71. doi: 10.1016/j.janxdis.2008.02.010

48. Szczurek L, Monin B, Gross JJ. The Stranger effect. Psychol Sci (2012) 23(10):1105-11. doi: 10.1177/0956797612445314
49. Kyriacou O, Easter A, Tchanturia K. Comparing views of patients, parents, and clinicians on emotions in anorexia. J Health Psychol (2009) 14(7):84354. doi: 10.1177/1359105309340977

50. Dapretto M, Davies MS, Pfeifer JH, Scott AA, Sigman M, Bookheimer SY, et al. Understanding emotions in others: mirror neuron dysfunction in children with autism spectrum disorders. Nat Neurosci (2006) 9(1):28-30. doi: $10.1038 / \mathrm{nn} 1611$

51. Evers K, Steyaert J, Noens I, Wagemans J. Reduced recognition of dynamic facial emotional expressions and emotion-specific response bias in children with an autism spectrum disorder. J Autism Dev Disord (2015) 45(6):177484. doi: 10.1007/s10803-014-2337-x

52. Uljarevic M, Hamilton A. Recognition of emotions in autism: a formal meta-analysis. J Autism Dev Disord (2013) 43(7):1517-26. doi: 10.1007/ s10803-012-1695-5

53. Doris E, Westwood H, Mandy W, Tchanturia K. A qualitative study of friendship in patients with anorexia nervosa and possible autism spectrum disorder. Psychology (2014) 5(11):1338. doi: 10.4236/psych.2014.511144

54. Westwood H, Lawrence V, Fleming C, Tchanturia K. Exploration of friendship experiences, before and after illness onset in females with anorexia nervosa: a qualitative study. PLoS One (2016) 11(9):e0163528. doi: 10.1371/journal.pone.0163528

55. Kanfiszer L, Davies F, Collins S. "I was just so different": the experiences of women diagnosed with an autism spectrum disorder in adulthood in relation to gender and social relationships. Autism (2017) 21(6):661-9. doi: $10.1177 / 1362361316687987$

56. Sedgewick F, Hill V, Pellicano E. "It's different for girls": gender differences in the friendships and conflict of autistic and neurotypical adolescents. Autism (2018). doi: 10.1177/1362361318794930

57. Sedgewick F, Hill V, Yates R, Pickering L, Pellicano E. Gender differences in the social motivation and friendship experiences of autistic and non-autistic adolescents. J Autism Dev Disord (2016) 46(4):1297-306. doi: 10.1007/ s10803-015-2669-1

58. Danner UN, Sanders N, Smeets PAM, van Meer F, Adan RAH, Hoek $\mathrm{HW}$, et al. Neuropsychological weaknesses in anorexia nervosa: setshifting, central coherence, and decision making in currently ill and recovered women. Int J Eat Disord (2012) 45(5):685-94. doi: 10.1002/ eat. 22007

59. Lopez C, Tchanturia K, Stahl D, Treasure J. Central coherence in eating disorders: a systematic review. Psychol Med (2008) 38(10):1393-404. doi: 10.1017/S0033291708003486

60. Burnette CP, Mundy PC, Meyer JA, Sutton SK, Vaughan AE, Charak D. Weak central coherence and its relations to theory of mind and anxiety in autism. J Autism Dev Disord (2005) 35(1):63-73. doi: 10.1007/ s10803-004-1035-5

61. Happé F, Frith U, Briskman J. Exploring the cognitive phenotype of autism: weak "central coherence" in parents and siblings of children with autism: I. Experimental tests. J Child Psychol Psychiatry (2001) 42(3):299-307. doi: $10.1017 /$ S0021963001006916

62. South M, Ozonoff S, Mcmahon WM. The relationship between executive functioning, central coherence, and repetitive behaviors in the highfunctioning autism spectrum. Autism (2007) 11(5):437-51. doi: 10.1177/ 1362361307079606

63. Tchanturia K, Adamson J, Leppanen J, Westwood H. Characteristics of autism spectrum disorder in anorexia nervosa: a naturalistic study in an inpatient treatment programme. Autism (2017) 23(1):123-30. doi: $10.1177 / 1362361317722431$

64. Grabhorn R, Stenner H, Stangier U, Kaufhold J. Social anxiety in anorexia and bulimia nervosa: the mediating role of shame. Clin Psychol Psychother (2006) 13(1):12-9. doi: 10.1002/cpp.463

65. Kerr-Gaffney J, Harrison A, Tchanturia K. Social anxiety in the eating disorders: a systematic review and meta-analysis. Psychol Med (2018) 48(15):2477-91. doi: 10.1017/S0033291718000752

66. Gillott A, Standen PJ. Levels of anxiety and sources of stress in adults with autism. J Intellect Disabil (2007) 11(4):359-70. doi: 10.1177/ 1744629507083585

67. Kim JA, Szatmari P, Bryson SE, Streiner DL, Wilson FJ. The prevalence of anxiety and mood problems among children with autism and Asperger syndrome. Autism (2000) 4(2):117-32. doi: 10.1177/1362361300004002002 
68. Kuusikko S, Pollock-Wurman R, Jussila K, Carter AS, Mattila M-L, Ebeling $\mathrm{H}$, et al. Social anxiety in high-functioning children and adolescents with autism and Asperger syndrome. J Autism Dev Disord (2008) 38(9):1697-709. doi: 10.1007/s10803-008-0555-9

69. Lavender JM, Mason TB, Utzinger LM, Wonderlich SA, Crosby RD, Engel SG, et al. Examining affect and perfectionism in relation to eating disorder symptoms among women with anorexia nervosa. Psychiatry Res (2016) 241:267-72. doi: 10.1016/j.psychres.2016.04.122

70. Limburg K, Watson HJ, Hagger MS, Egan SJ. The relationship between perfectionism and psychopathology: a meta-analysis. J Clin Psychol (2017) 73(10):1301-26. doi: 10.1002/jclp.22435

71. Lloyd S, Yiend J, Schmidt U, Tchanturia K. Perfectionism in anorexia nervosa: novel performance based evidence. PLoS One (2014) 9(10):e111697. doi: 10.1371/journal.pone.0111697

72. Tchanturia K, Smith E, Weineck F, Fidanboylu E, Kern N, Treasure J, et al. Exploring autistic traits in anorexia: a clinical study. Mol Autism (2013) 4(1):44. doi: 10.1186/2040-2392-4-44
73. Booth T, Murray AL, McKenzie K, Kuenssberg R, O’Donnell M, Burnett H. Brief Report: an evaluation of the AQ-10 as a brief screening instrument for ASD in adults. J Autism Dev Disord (2013) 43(12):2997-3000. doi: 10.1007/ s10803-013-1844-5

74. Sappok T, Heinrich M, Underwood L. Screening tools for autism spectrum disorders. Adv Autism (2015) 1(1):12-29. doi: 10.1108/AIA-03-2015-0001

Conflict of Interest Statement: The authors declare that the research was conducted in the absence of any commercial or financial relationships that could be construed as a potential conflict of interest.

Copyright (C) 2019 Sedgewick, Leppanen, Goh, Hayward, Happe and Tchanturia. This is an open-access article distributed under the terms of the Creative Commons Attribution License (CC BY). The use, distribution or reproduction in other forums is permitted, provided the original author(s) and the copyright owner(s) are credited and that the original publication in this journal is cited, in accordance with accepted academic practice. No use, distribution or reproduction is permitted which does not comply with these terms. 